\title{
New data for the comprehension of the LSND anomaly
}

\author{
Alexey ZHEMCHUGOV* (for the HARP-CDP group ${ }^{\dagger}$ ) \\ Joint Institute for Nuclear Research \\ E-mail: zhemchugovejinr.ru
}

The so-called 'LSND anomaly', a $3.8 \sigma$ excess of $\bar{v}_{e}$ events, resulted in many theoretical speculations. The interpretation by the LSND authors that the anomaly originates from $\bar{v}_{\mu} \rightarrow \bar{v}_{e}$ oscillation, is inconsistent with the recent findings of the MiniBooNE Collaboration. We present a critical assessment of the prediction of the neutrino fluxes that were used in the analysis of the LSND experimental data, which possibly can shed light on the anomaly. Our appraisal is based on the pion spectra measured with the HARP large-angle spectrometer under conditions that closely reproduce the LSND situation: a proton beam with $800 \mathrm{MeV}$ kinetic energy bombarding water and copper targets.

European Physical Society Europhysics Conference on High Energy Physics, EPS-HEP 2009,

July 16 - 222009

Krakow, Poland

\footnotetext{
${ }^{*}$ Speaker.

${ }^{\dagger}$ The members of the HARP-CDP group are: A. Bolshakova, I. Boyko, G. Chelkov, D. Dedovich, A. Elagin, M. Gostkin, S. Grishin, A. Guskov, Z. Kroumchtein, Yu. Nefedov, K. Nikolaev and A. Zhemchugov from the Joint Institute for Nuclear Research, Dubna, Russian Federation; F. Dydak and J. Wotschack from CERN, Geneva, Switzerland; A. De Min from the Politecnico di Milano and INFN, Sezione di Milano-Bicocca, Milan, Italy; and V. Ammosov, V. Gapienko, V. Koreshev, A. Semak, Yu. Sviridov, E. Usenko and V. Zaets from the Institute of High Energy Physics, Protvino, Russian Federation.
} 


\section{Introduction}

The LSND experiment reported an anomalous $3.8 \sigma$ excess of $\bar{v}_{e}$, interpreted as $\bar{v}_{\mu} \rightarrow \bar{v}_{e}$ oscillations with $\Delta m^{2} \approx 1 \mathrm{eV}^{2}$ [1]. One explanation of the LSND signal is an underestimate of the $\pi^{-}$production assumed in the calculation of the background neutrino flux in the LSND analysis. The experimental measurement of pion production by $800 \mathrm{MeV}$ protons on different targets is a crucial test of this explanation.

\section{Detector characteristics and performance}

The HARP experiment at the CERN PS took data in 2002 with proton and pion beams of 1.5 $\mathrm{GeV} / c$ momentum impinging on water, copper, lead and tantalum targets. The detector comprised a forward spectrometer and a large-angle spectrometer.

The work reported here is exclusively concerned with data from the HARP large-angle spectrometer $[2,3]$. The latter consists of a cylindrical TPC that measures $p_{\mathrm{T}}$ and the polar angle $\theta$ of tracks, and their specific ionization $\mathrm{d} E / \mathrm{d} x$. Timing RPCs that surround the TPC measure time of flight with a view to complementing and corroborating particle identification. The main detector performance parameters are summarized in Table 1.

\begin{tabular}{|l|l|}
\hline TPC & RPCs \\
\hline \hline$\sigma\left(1 / p_{\mathrm{T}}\right) \sim 0.20-0.25(\mathrm{GeV} / c)^{-1}$ & Intrinsic efficiency 98\% \\
$\sigma(\theta) \sim 9 \mathrm{mrad}$ & $\sigma(\mathrm{TOF}) \sim 175 \mathrm{ps}$ \\
$\sigma(\mathrm{d} E / \mathrm{d} x) \sim 0.16$ & \\
\hline
\end{tabular}

Table 1: Main performance parameters of the HARP large angle spectrometer

\section{HARP-CDP measurements versus LSND Monte-Carlo simulation}

We have measured the $\pi^{-} / \pi^{+}$ratio on a $60 \mathrm{~cm}$ long $\mathrm{H}_{2} \mathrm{O}$ target and inclusive cross-section of $\pi^{+}$and $\pi^{-}$production on thin (5\% of $\lambda_{\text {abs }}$ ) water, copper, lead and tantalum targets for incoming protons of $1.5 \mathrm{GeV} / c$ momentum. The results obtained are compared with the parametrization of pion production used in the LSND Monte-Carlo simulation of the neutrino flux emanating from the LAMPF beam stop [4]. The comparison is shown in Figs 1 and 2.

The measured $\pi^{-} / \pi^{+}$ratio on water is smaller than the $\pi^{-} / \pi^{+}$ratio utilized in the LSND analysis. For the copper target and for the heavy materials lead and tantalum, the situation tends to reverse. For an appreciation of the overall effect in terms of the neutrino flux, the precise knowledge of the contributions from different materials in the LAMPF beam stop to the overall neutrino flux is of crucial importance. This will be addressed in a forthcoming paper, for which the results presented will serve as input. 

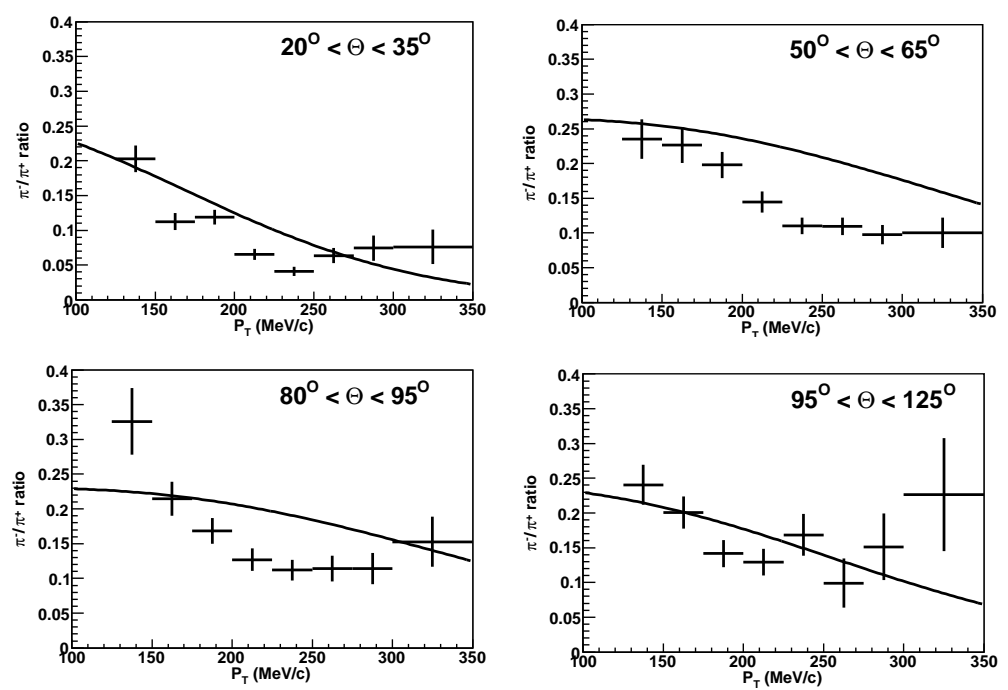

Figure 1: $\pi^{-} / \pi^{+}$ratio on water for four polar angle ranges. The curves show the parametrization used in the LSND analysis. The result is preliminary.
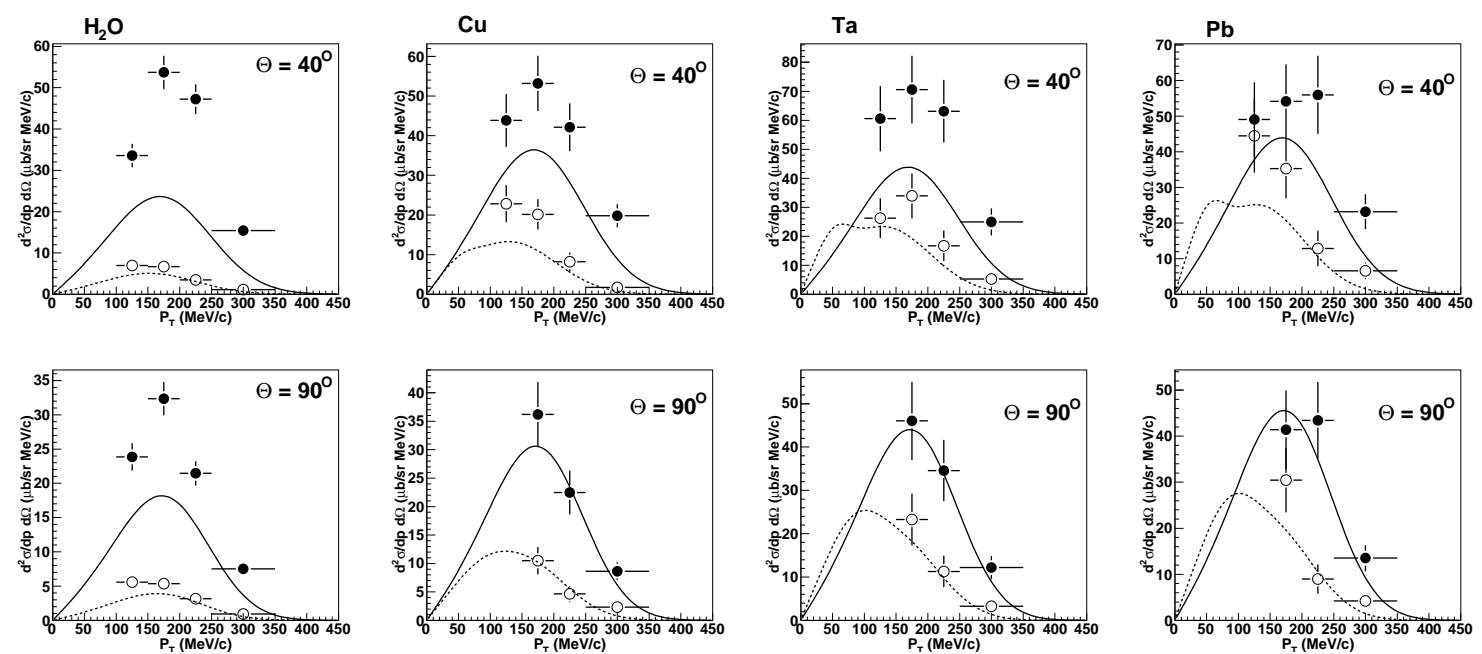

Figure 2: Cross-sections of the production of secondary $\pi^{+}$(black circles) and $\pi^{-}$(open circles) on water, copper, tantalum and lead (from left to right), compared with the LSND parametrization (the full line refers to $\pi^{+}$, and the broken line refers to $\pi^{-}$). The results are preliminary.

\section{References}

[1] A. Aguilar et al., Phys. Rev. D64 (2001) 112007

[2] V. Ammosov et al., Nucl. Instrum. Methods Phys. Res. A588 (2008) 294

[3] V. Ammosov et al., Nucl. Instrum. Methods Phys. Res. A578 (2007) 119

[4] We thank Myungkee Sung for providing the LSND parametrization; the program code is available at http://hep.phys.lsu.edu/sung/lsnd/beammc/index.html 